\title{
Single Cas9 nickase induced generation of NRAMP1 knockin cattle with reduced off- target effects
}

\author{
Yuanpeng Gao ${ }^{1,2 \dagger}$, Haibo Wu ${ }^{1,2 \dagger}$, Yongsheng Wang ${ }^{1,2+}$, Xin $\mathrm{Liu}^{1,2}$, Linlin Chen², Qian Li ${ }^{1,2}$, Chenchen Cui ${ }^{1,2}$,
} Xu Liu ${ }^{1,2}$, Jingcheng Zhang ${ }^{2}$ and Yong Zhang ${ }^{1,2^{*}}$

\begin{abstract}
Background: The CRISPR-Cas9 system is a widely utilized platform for transgenic animal production in various species, although its off-target effects should be addressed. Several applications of this tool have been proposed in model animals but remain insufficient for transgenic livestock production.

Results: Here, we report the first application of single Cas9 nickase (Cas9n) to induce gene insertion at a selected locus in cattle. We identify the main binding sites of a catalytically inactive Cas9 (dCas9) protein in bovine fetal fibroblast cells (BFFs) with chromatin immunoprecipitation sequencing (ChIP-seq). Subsequently, we demonstrate that a single Cas9n-induced single-strand break can stimulate the insertion of the natural resistance-associated macrophage protein-1 (NRAMP1) gene with reduced, but still considerable, off-target effects. Through somatic cell nuclear transfer, we finally obtain transgenic cattle with increased resistance to tuberculosis.
\end{abstract}

Conclusions: Our results contribute to the development of CRISPR-Cas9 system for agriculture applications.

Keywords: CRISPR-Cas9, Off-target, Homologous recombination, Chromatin immunoprecipitation sequencing (ChIP-seq), Nickase, Single-strand break, Tuberculosis

\section{Background}

Homologous recombination (HR) has been widely applied to facilitate the exchange of DNA sequences between a targeted chromosomal locus and a homologous template containing the desired change [1-3]. HR is inefficient in many cell types and thus a site-specific double-strand break (DSB) is often introduced by sitespecific homing endonucleases or artificial endonucleases, such as zinc finger and TALE nucleases. These endonucleases are introduced in the vicinity of the targeted genomic locus to stimulate subsequent DNA repair $[4,5]$. Most recently, the type II bacterial clustered, regularly interspaced, short palindromic repeats (CRISPR)-associated protein 9 (Cas9) system has come to represent an efficient tool for the further

\footnotetext{
*Correspondence: zhy1956@263.net

${ }^{\dagger}$ Equal contributors

${ }^{1}$ College of Veterinary Medicine, Northwest A\&F University, Yangling 712100, Shaanxi, China

${ }^{2}$ Key Laboratory of Animal Biotechnology, Ministry of Agriculture, Northwest A\&F University, Yangling 712100, Shaanxi, China
}

advancement of gene targeting strategies [6-10] due to the simplicity of targeting any locus for cleavage with a single protein and a programmable single-guide RNA (sgRNA).

A site-specific DSB can efficiently stimulate HR by approximately 10,000-fold [11, 12]. Nonetheless, the competing non-homologous end-joining (NHEJ) pathway for DSB repair is often favored and frequently leads to small insertions/deletions (indels) or chromosomal rearrangements, particularly in mammalian cells [13, 14]. Thus, this repair pathway causes a major safety problem for gene targeting strategies, particularly for gene therapy and transgenic animal production $[15,16]$. A number of studies have focused on enhancing the efficiency, specificity, and versatility of the CRISPR/Cas9 system [14, 17, 18], but reports of large functional gene insertions in mammalian genome editing are limited.

In the current study, chromatin immunoprecipitation (ChIP) was performed on a catalytically inactive double mutant (D10A and H840A) Cas9 (dCas9) [7, 19] for 
protein binding site detection in bovine fetal fibroblast cells (BFFs) and followed by sequencing (ChIP-seq). According to findings by others $[6,20]$, the single Cas9 nickase (Cas9n)-mediated single-strand break (SSB) has the potential to generally avoid the NHEJ repair pathway. Consequently, we determined the feasibility of using Cas9n-induced SSB to stimulate homology-directed repair (HDR) and therefore provided a safe alternative for gene insertion and transgenic animal generation. After a systematic selection of target sites and transgenic colonies, we successfully obtained nine exogenous natural resistance-associated macrophage protein-1 (NRAMP1) gene-inserted cows through the Cas9n strategy. Moreover, reduced off-target effects were detected both in the transgenic BFFs and cattle. We further demonstrated that Cas9n-mediated NRAMP1 insertion provided the cattle with increased resistance to tuberculosis.

\section{Results}

Prediction and selection of the targeting locus and sgRNAs

Considering the potential synergistic effects of neighboring genes, we used an intergenic region between the fascin actin-bundling protein 1 gene (FSCN1) and the actin beta gene $(A C T B)$ on chromosome 25 (positions $40,631,870-40,632,430 ; 561 \mathrm{bp}$ long) as the potential gene target region (Additional file 1: Supplemental dataset S1). Housekeeping genes in the FSCN1-ACTB (FA) locus (Fig. 1a) have relatively steady expression levels across various tissues and thus exogenous gene silencing resulting from chromatin inactivation might be avoided. We used the open-source website ZiFiT [21-23] (http:// zifit.partners.org/ZiFiT/) and identified a total of 80 target sites in this locus. These sites either ended with NGG or started with $\mathrm{CCN}$ on the reverse strand (i.e. the
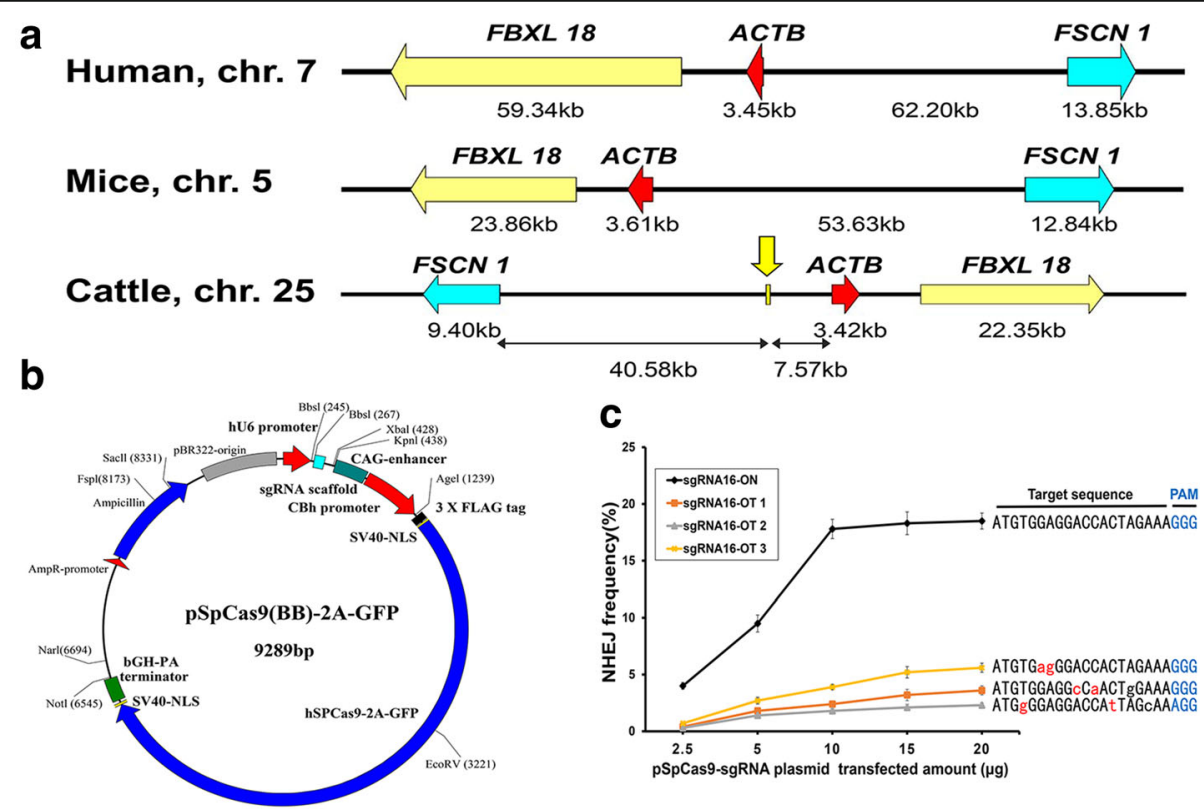

d
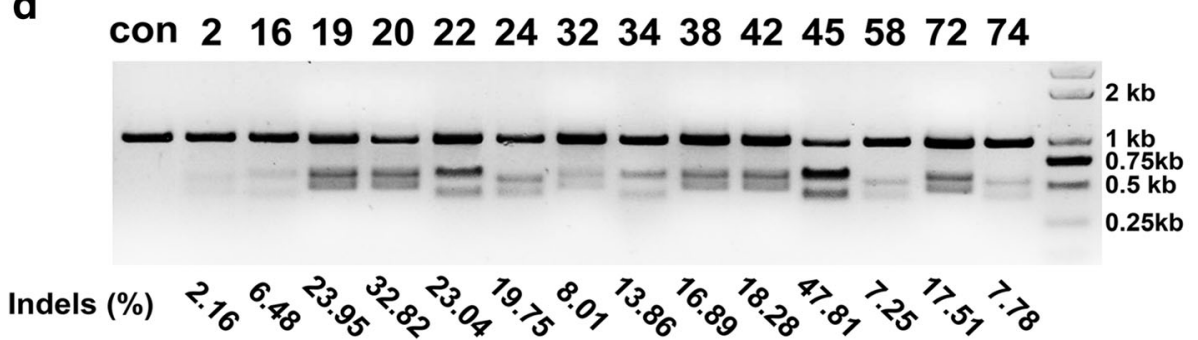

Fig. 1 F-A locus and target site selection. a The molecular structures of the human, mouse, and cattle F-A loci were highly conserved. b Schematic representation of the plasmid encoding both hSpCas9 expression and sgRNA transcription. Different sgRNAs were cloned for the corresponding target sites through the two neighboring Bbsl restriction enzyme sites. c Concentration-dependent cleavage activity and the amount of pSpCas9 DNA transfected. The three potential off-target sites were selected with Cas-OFFinder based on fewer than three mismatches and the NHEJ frequency was determined by a Surveyor nuclease assay. $\mathbf{d}$ Cleavage efficiencies of the Cas9 protein at the selected target sites. "Con" represents the DNA isolated from BFFs transfected with Cas9 only (without sgRNA). The degree of cleavage was quantified with Surveyor nuclease assays and ImageJ (http://imagej.net). The formula for estimating the indel rate is provided in the "Methods" section 
protospacer adjacent motif (PAM)) [24] (Additional file 2: Supplemental dataset S2).

Regardless of the significant difference in the nucleotide number (varying from 5 to 12) of the seed region $[14,17,25,26]$, the PAM-proximal seed region of the guide sequence contributes more to the overall specificity of Cas9-mediated DNA cleavage and tolerates fewer mismatches than the PAM-distal sequences [19, 27, 28]. To implement the CRISPR/Cas9 system for cattle genome editing, we developed an evaluation model for the selection of sgRNAs. This evaluation model accounts for both the number of mismatches through an effective web-based off-target prediction tool termed CasOFFinder [29] (http://www.rgenome.net/cas-offinder) and the flexible relevance between the mismatch location and the seed region (Additional file 2: Supplemental dataset S2). As an alternative to this evaluation model, we also presented most of the predicted off-targets in detail. For example, the visualized possible off-targetenriched zones in the hypothetical 12-, 8-, and 5nucleotide seed regions are presented as well as the copy number of the seed + NGG sequence at the genome level. The detailed data are available in Additional file 2: Supplemental dataset S2 and Additional file 3: Figure S1. Finally, we selected 14 potential target sites for subsequent efficiency detection.

\section{Construction of Cas9 target plasmids and cleavage efficiency detection}

To avoid interference from the relative ratio discrepancy of the hspCas9 protein and sgRNA, we chose the widely used all-in-one pSpCas9 (BB)-2A-GFP plasmid (PX458, Addgene plasmid \#48138) [30] as the basic target plasmid (Fig. 1b). The primers used to clone each sgRNA are available in Additional file 3: Table S1. The off-target cleavage of Cas9 is highly sensitive to the transfected amount of plasmid [25, 27, 30]; hence, we randomly selected the target site 16 and carefully titrated the amount of the transfected reformed plasmid pSpCas9sgRNA16. When the transfected DNA was increased to $10 \mu \mathrm{g}$, the frequency of disruption at the target site reached a peak, whereas the disruptions of three putative off-target sites still occurred at relatively low levels (Fig. 1c). Thus, this concentration was optimal.

Subsequently, the DNA cleavage efficiencies in the BFFs at all 14 target sites were determined via Surveyor nuclease assays [31]. The results indicated that the majority $(10 / 14)$ of the cleavage efficiencies $\left(f_{\text {cut }}\right)$ were in the range of $13.97-40.77 \%$ and the corresponding indel rate was in the range of $7.25-23.04 \%$ (Fig. 1d and Additional file 3: Table S2). Notably, the indel percentages in sgRNA20 and sgRNA45 reached $32.82 \%$ and $47.18 \%$, respectively. We further amplified the genomic target loci from the pSpCas9-sgRNA22- and pSpCas9-
sgRNA45-transfected BFFs via polymerase chain reaction (PCR) and TA cloning. Subsequently, totals of 182 and 179 transformed Escherichia coli bacterial colonies were randomly selected for Sanger sequencing to confirm the presence of Cas9 nuclease-induced indels at the targeted locus and these colonies had indel rates of $21.43 \%$ and $41.90 \%$, respectively (representative sequences are provided in Additional file 3: Figure S2). These results agreed with those from the Surveyor nuclease assays. Thus, these detection efforts provided target sites with noticeably different cutting efficiencies for subsequent experiments.

\section{Genome-wide binding of the sgRNA-dCas9 in BFFs}

To gain insight into the targeting specificity and the extent of the off-target effects of CRISPR/Cas9 system in the bovine genome, we performed ChIP-seq for dCas9 protein binding site detection in BFFs. A total of four target sites $(2,20,22$, and 45$)$ with typical cleavage efficiencies were used and the corresponding sgRNAs were cloned into the $3 \times$ FLAG-tagged dCas 9 expression vector (Fig. 1b). After the transfection of these plasmids into the BFFs for $48 \mathrm{~h}$, ChIP was performed following immunoprecipitated dCas9-associated DNA sequencing on a single lane of the Illumina HiSeq 2500 platform (Fig. 2a). The clean reads were then aligned to the Bos taurus genome sequence (version: Btau_4.6.1) using the BWA program [32].

Visually strong peaks (200-500 bp) were observed at the intended target sites of each sgRNA (Fig. 2b) and large numbers of common peaks were also detected among the different sgRNAs (including the dCas9-only group). These observations are consistent with those of previous studies $[26,28]$ that have identified such peaks as false positives due to sequencing biases, repeat-rich sequences, and GG/CC-rich motifs in a particular chromosomal region. To focus on the dCas9-binding sites that are specific for particular sgRNAs, we applied pair-wise peak calling with model-based analysis of ChIP-seq (MACS) [33] between each sgRNA sample and the other groups (including the dCas9-only control). Hence, only the enriched peaks in an individual group were retained for the remaining analyses.

Because both the on-target (Fig. 2b) and potential offtarget sites (Fig. 2c) occurred primarily in the centers of the binding peaks, we computationally identified the 20bp-long potential recognition sequences. These sites both ended with PAM and aligned most effectively with the sgRNA-guiding sequence. Subsequently, the top 15 off-target sites of each sgRNA group were ranked according to the ChIP-seq binding density (peak fold enrichment) as illustrated in Fig. 3 and Additional file 3: Figure S3. The greatest density of Cas9 binding was located at the on-target site in all four sgRNA groups, but 

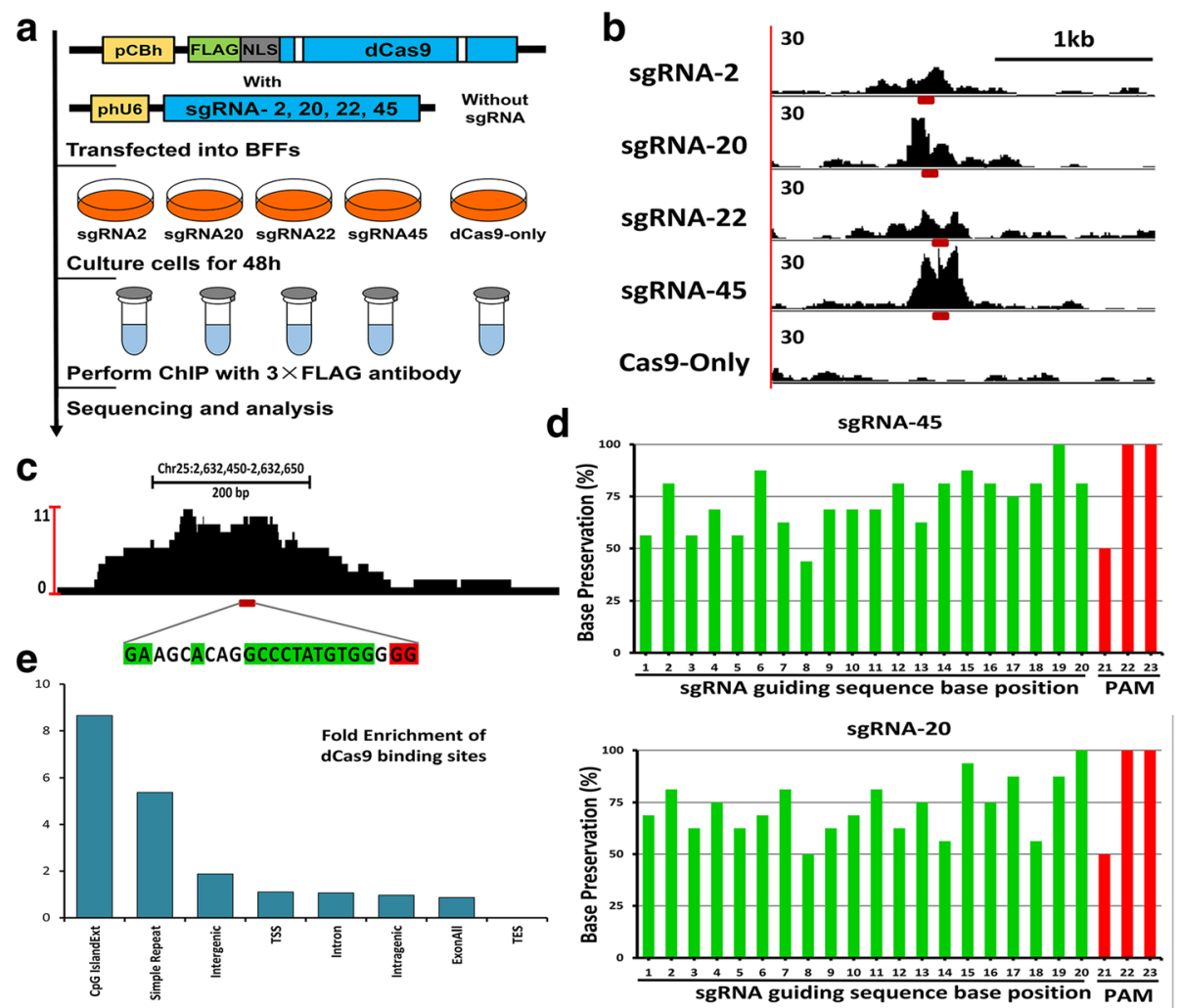

Fig. 2 Process and characteristics of dCas9 binding in BFFs. a Schematic representation of the dCas9 ChIP-seq approach applied to the BFFs. The four $3 \times$ FLAG-tagged Cas9-encoding plasmids with different sgRNAs were considered different experimental groups (left) and the same plasmid but without sgRNA was used for the dCas9-only control (right). b Visualization of the ChIP-seq peaks (normalized read counts) revealed that they were localized around four on-target sites and the control. The red dashes under each peak indicate the designed target sites. $\mathbf{c}$ Visualization of the ChIP-seq peak of one typical off-target region. The location of this region is shown above the peak. Bases matching the sgRNA guiding sequences and PAM sequences at the off-target sites are highlighted in green and red, respectively. $\mathbf{d}$ Percentages of preserved bases at the main off-target sites compared with the guiding sequences of sgRNA45 (above) and sgRNA 20 (below). e Performance of dCas9 in binding to the chromatin structure of the off-target binding site. "TSS" represents the $1 \mathrm{~kb}$ region centered on the transcription start site region; "TES" represents the same range of the transcription end site

many of the peaks at the off-target sites exhibited high signal intensity. This observation demonstrated the existence of substantial off-target sites for the dCas9sgRNA complexes that bound in the BFFs and might address the necessity to confirm the actual off-target effects to avoid unexpected gene modifications. Regarding sgRNA2, some of the off-target peaks were greater than the on-target peak (Additional file 3: Figure S3), which might have resulted from the additive effect of multiple potential binding sites in the same peak [28].

\section{Characteristics of dCas9 binding in BFFs}

Additionally, we compared the base compositions of the main off-target sites with the on-target sequence (Fig. $2 \mathrm{~d}$ and Additional file 3: Figure S4) to assess the presence and composition of the "seed sequence." This sequence was defined as the conserved sequence next to the PAM and was less tolerant of mismatches in the sgRNA. As expected, a higher base preservation rate was observed in the PAM-proximal position than in the PAM-distal region. Using sgRNA $45(\mathrm{n}=3562$ sites $)$ as an illustration, eight of the ten PAM-proximal bases were preserved in $75 \%$ of the off-target sites and only two bases were conserved in the PAM-distal ten bases. Similar but smaller magnitude trends were observed for the sgRNAs 20 and 22. Nevertheless, no obvious base preservation was observed in the proximal or distal regions of sgRNA 2. In accordance with the conclusion from previous studies, we confirmed that all positions were preserved in the sgRNA-guiding sequence, but the PAM-proximal seed region tolerated fewer mismatches and contributed more to the binding specificity than the remaining regions in the BFFs.

To detect the performance of dCas9 binding to the chromatin structure, the potential off-target sites were analyzed (Fig. 2e) based on the UCSC genome annotation. Considering the large number of common peaks in the repeat-rich sequences and the GG/CC-rich motifs (described above), we also considered the strong enrichment at CpG islands and simple repeats as false positives 


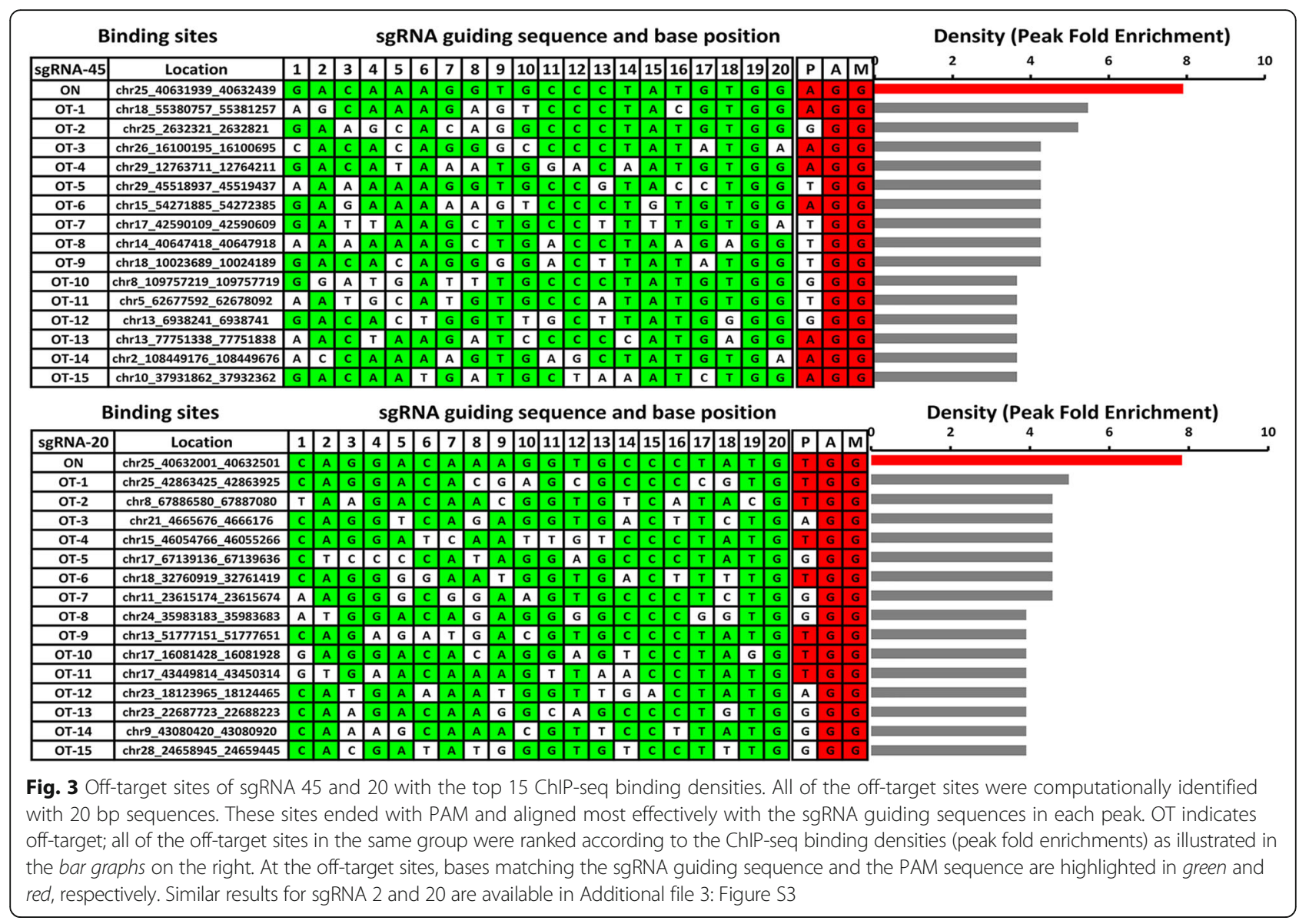

along with the results from the imperfect pair-wise peak calling with MACS. Interestingly, an approximately 40fold enrichment was observed in the microsatellite sequence motifs, but this enrichment was primarily eliminated after the pair-wise peak calling process. The greater enrichment values in the transcription start site (TSS) regions compared with the transcription end site (TES) regions has previously been reported [28]. This result suggested that the chromatin structure might influence dCas9 binding. The substantial enrichment of off-target binding in the intergenic regions of the BFFs might also support the target locus selection of this study.

The ChIP enrichment level correlates well with the DNA binding intensity and the frequency of interaction [34], but the correlation between Cas9 binding and DNA cleavage remains to be tested. To corroborate the practical indel frequencies induced by the Cas9 nuclease at the ChIP-seq designated off-target sites, we again transfected reformed plasmids encoding wild-type (WT) Cas9 or Cas9n with sgRNAs 2, 20, 22, and 45 into BFFs. Subsequently, the peak-covered sequences were amplified by PCR and deep sequenced (the primers are provided in Additional file 3: Table S3). The plasmid encoding WT Cas9 without sgRNA was also used as a control at the same sites. Detailed calculations of the indel rates at the 60 main off-target sites and four ontarget sites are provided in Additional file 3: Table S4. As expected, high indel rates were observed at the four on-target sites in the WT Cas9 group. Moreover, 13 of the 60 off-target sites (21.7\%) exhibited significantly greater indel rates than the control $(P<0.05$, Fisher's exact test) and four of these sites belonged to the sgRNAs 20 and 45 .

Similar indel frequencies were observed in the single Cas9n group at nearly all sites compared with the control. In addition, the single Cas9n group has low indel rates at the target site compared with the WT Cas9 group (Additional file 3: Table S4). Based on the substantial indel frequency detection at the off-target sites, we hypothesized that single Cas9n-mediated SSB can generally avoid the NHEJ repair pathway.

\section{Single Cas9n succeeded in inducing desired HDR with considerable efficiency}

To further evaluate the Cas9n-induced HDR recombination efficiency, we selected target sites 20, 45, and two paired target sites, i.e. $2+19$ and $16+34$, for the subsequent 
experiments. In the double-nicking strategy, two highly active sgRNAs in sufficient proximity (selected through ZiFit) are required to design an efficient nickase pair $[9,20]$. We designed two donor plasmids as HDR repair templates in which an EcoR-I restriction site was flanked by the paired homology arms for the above four groups (Fig. 4a).

WT Cas9, single Cas9n, or paired Cas9ns recombinant plasmid(s) were respectively co-transfected with the donor plasmid into the BFFs. The genomic DNA was extracted for targeted-locus PCR-amplification $72 \mathrm{~h}$ later and the subsequent EcoR-I tag digestion assays were showed in Fig. 4b. Compared with the WT Cas9 groups, all of the single Cas9n-induced HDRs occurred at perceptibly low frequencies $(60-80 \%$ reductions), which indicated a decrease in the HDR efficiency at the same target site (Fig. 4b). We also observed that the HDR recombination rates of the single Cas9n at the target sites $20(4.5 \%)$ and $45(8.3 \%)$ were comparable to those of the paired Cas9n-2 + 19 (4.2\%) and Cas9n-16+34 (5.3\%) group.

Taken together, these results indicated that single Cas9n-mediated SSB can successfully induce HDR recombination at a systematically selected target site with reduced but still considerable efficiency.

\section{Selection of the transgene}

To address one of the major diseases in cattle and minimize immunological disruptions, we focused on endogenous functional genes associated with innate immunity for the transgene. The first identified tuberculosis-susceptible candidate [35], i.e. the NRAMP1 gene, which has been renamed as the solute carrier family $11 \mathrm{~A}$ member 1 gene (SLC11A1), is associated with innate resistance to intracellular pathogens, such as Mycobacterium, Leishmania, Salmonella, and Brucella [36-38]. The expression of NRAMP1, which exclusively occurs in macrophages and other dedicated phagocytes, is upregulated by cytokines and induces the production of nitric oxide (NO) in addition to other proinflammatory responses [39].

In the present work, the bovine NRAMP1 cDNA sequence was amplified from the peripheral blood of Holstein-Friesian cows (the sequence is available in Additional file 1: Supplemental Dataset S1). This
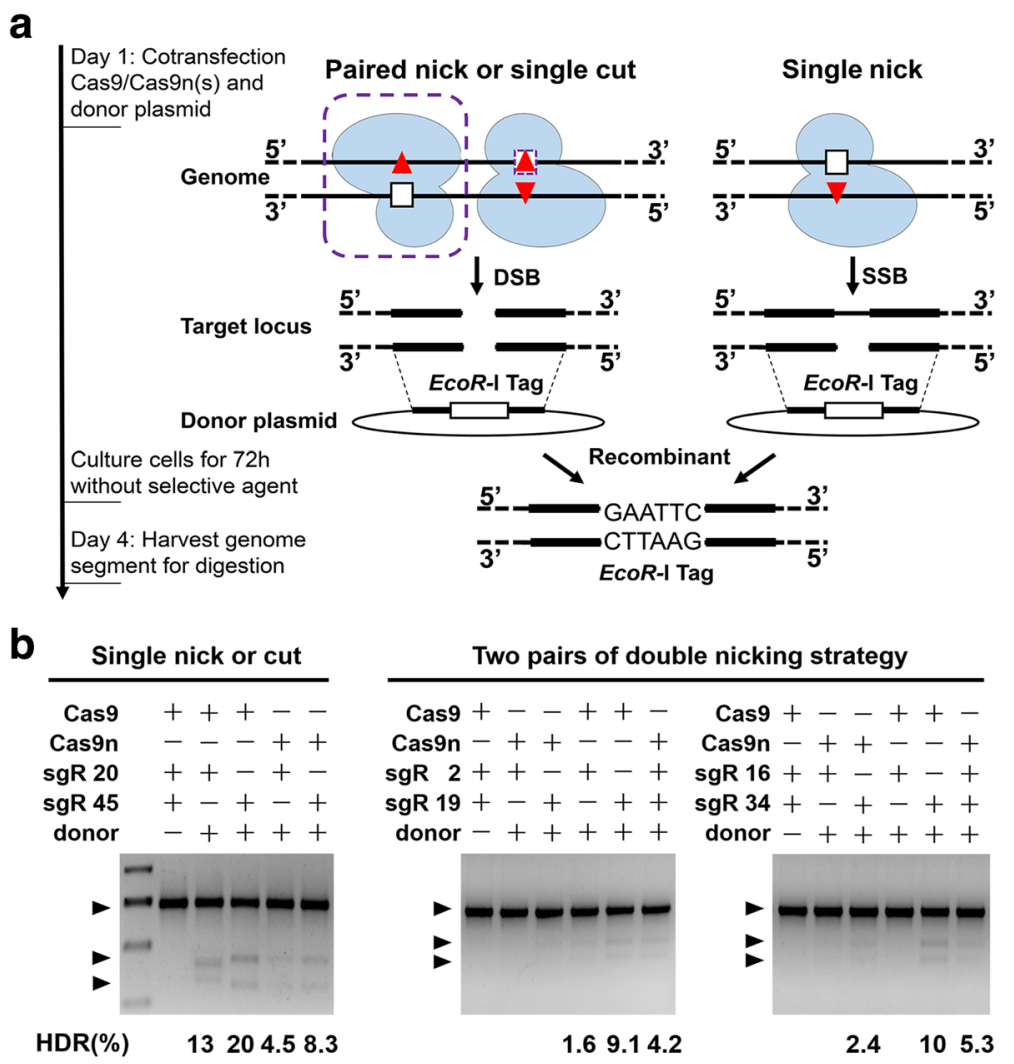

Fig. 4 Single Cas9n stimulated HDR at the F-A locus. a Experiential outline and schematic of the HDR process. The designed donor plasmid was recombined with the genome through single Cas9-induced DSB (left, without the purple frames), paired Cas9n-induced DSB (left, with the purple frames), and single Cas9n-induced SSB (right). $\mathbf{b}$ HDR frequency measurements of the different targeting strategies based on restriction enzyme tag integration. The specific numbers below each lane were calculated with the relative intensities of digested bands and the undigested band 
sequence displayed 99\% identity with the published sequence (NCBI Gene ID: 282470), i.e. there were only two nucleotide variations. The only non-synonymous nucleotide substitution occurred in codon 49 (ACA to GCA) and led to a threonine-to-alanine residue change that represents a functionally irrelevant single nucleotide polymorphism (SNP) based on previous work [40]. Moreover, the overexpression of bovine NRAMP1 can reduce the multiplication of Mycobacterium bovis (M. bovis) in the murine RAW264.7 macrophage cell line after infection via a cfu test (Additional file 3: Figure S5, $96 \mathrm{~h}: 325 \% \pm 21 \%$ versus $136 \% \pm 24 \%, P=0.0017)$. This cell line carries the NRAMP1 G169D allele and therefore displays a functional NRAMP1 deficiency [36]. Consequently, we decided to add a NRAMP1 gene to the specific locus above in the bovine genome.

\section{Single Cas9n mediates a site-specific NRAMP1 insertion in} BFFs

The gene-targeting donor vectors pNRAMP1-eGFPP2A-Puro-1/2 (Fig. 5a) were constructed with the same element but different homology arms for the corresponding target sites. The sequences are provided in Additional file 1: Supplemental Dataset S1. We cloned the Holstein NRAMP1 5' flanking region $(-1748$ to +73$)$ as an original promoter sequence to direct NRAMP1 expression at a level comparable to that of the natural gene activity. Because the selected markers are driven by an $\mathrm{EF} 1 \alpha$ promoter, the puromycin gene and enhanced green fluorescent protein were fused to the porcine teschovirus-1 2A (P2A) peptide sequence [41]. Subsequently, two LoxPs in the same orientation were added to each side to remove the markers when required [42]. We transfected the donor vector, along with an expression
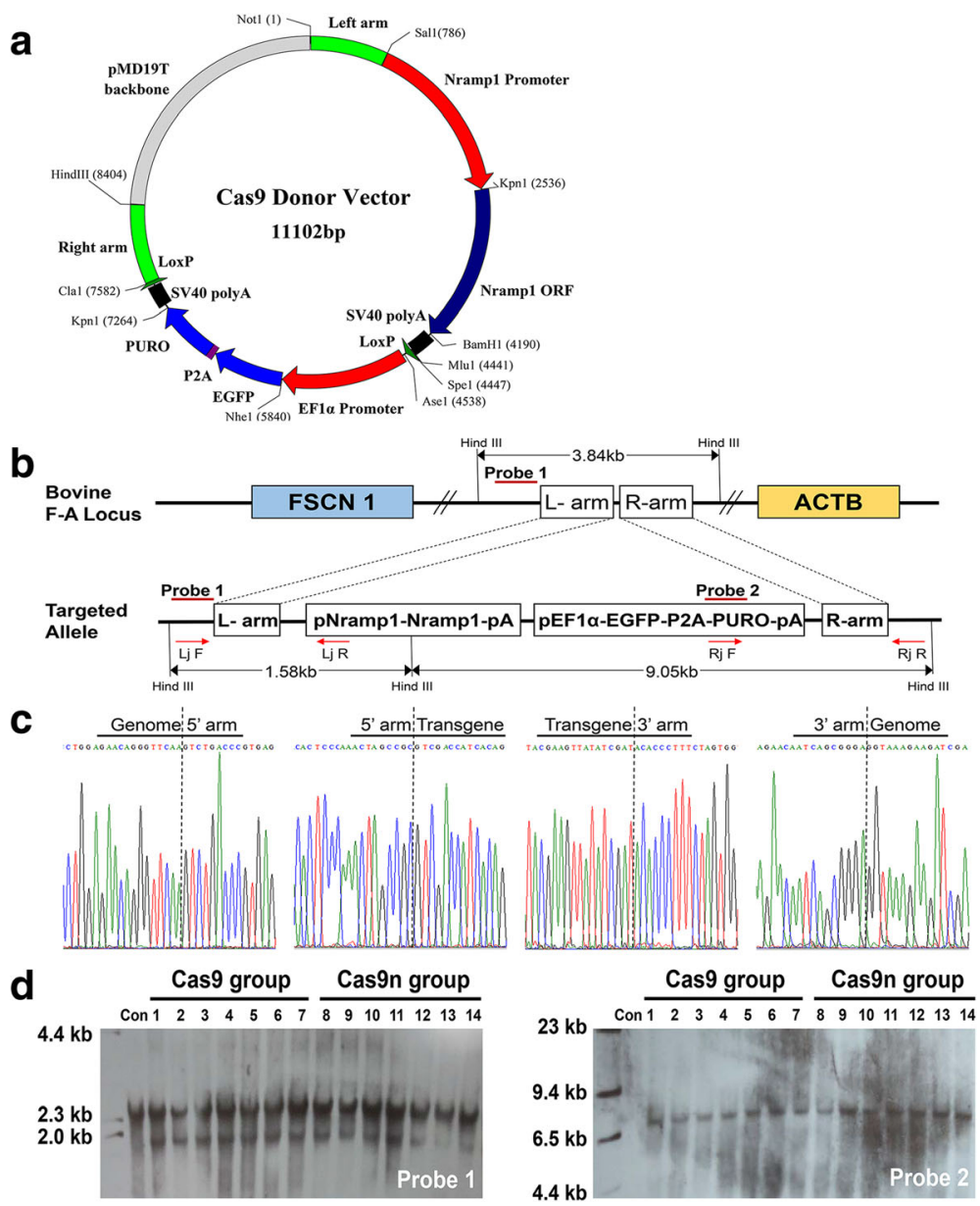

Fig. 5 Insertion and selection of the NRAMP1 transgenic colony using single Cas9 or Cas9n. a Schematic representation of the gene-targeting donor vector. b Schematic overview of the screening of the individual colonies. Lj F and Rj R were the primers for the regions outside the homologous arms, and Lj R and Rj F were the primers for the donor vector region. Southern blot probes are shown as red lines and Hind III digestion was used in the southern blot analysis. c Sanger sequencing confirming the precise insertion of the exogenous DNA. $\mathbf{d}$ Southern blot analysis of the donor cells used for SCNT. Non-transfected BFFs were used for negative controls. A $1.58 \mathrm{~kb}$ band resulting from the targeted insertion of the NRAMP1 cassette was detected in addition to the $3.84 \mathrm{~kb}$ band from the endogenous F-A locus allele when probe 1 was used. A $9.05 \mathrm{~kb}$ targeted band was also detected with probe 2 
plasmid encoding WT cas9, single cas9n, or paired cas9n, to introduce a $\mathrm{DSB} / \mathrm{SSB}$ and the further insertion of NRAMP1 at four target sites $(20,45,2+19$, and $16+34)$ in the BFFs (Additional file 3: Table S5). Early-passage (P2 or P3) primary BFFs were obtained from female HolsteinFriesian dairy cows and used for targeting.

Following 8-10 days of puromycin $(2 \mu \mathrm{g} / \mathrm{mL})$ selection, firmly transfected colonies were trypsinized and screened by $5^{\prime}$ junction $(1.58 \mathrm{~kb})$ and $3^{\prime}$ junction $(1.98 \mathrm{~kb})$ PCR. The product should contain both the insert and internal genome sequences to confirm that stable genetic cell modification occurred at the intended specific site (Fig. 5b, c, and Additional file 3: Table S5). Representative PCR results are provided in Additional file 3: Figure S6. Statistical tests revealed that 34.55\% $(57 / 165)$ of the puromycin-resistant colonies contained cells that were correctly targeted at site 45 by WT Cas 9 , whereas the positive recombination rate for single Cas9n at the same site was $18.11 \%(69 / 381)$. The weakened positive recombination rate agreed with the HDR efficiency detected above (Fig. 4b). Based on our previous studies [43,44], a positive recombination rate of $18.11 \%$ should be sufficient for gene editing.

To further evaluate the ploidy of transgenic cassette in the clones that passed two rounds of junction PCR, we designed two probes for southern blot analyses (Fig. 5d).
The $1.58 \mathrm{~kb}$ band (probe 1) resulting from the NRAMP1 cassette insertion and the single $9.05 \mathrm{~kb}$ targeted band (probe 2) suggested site-specific integration. The $3.84 \mathrm{~kb}$ band (probe 1) from the endogenous F-A locus allele suggested that the tested colonies were heterozygous and exhibited one normal chromosome. Constitution of the positive cell colonies generated by different types of Cas9 at the target site 45 were available in Additional file 3: Table S6. After confirming the successful insertion, karyotype analysis of each heterozygous colony was performed (a typical and representative karyotype is illustrated in Additional file 3: Figure S7).

Finally, a total of 113 heterozygous colonies that were edited at site 45 and exhibited normal karyotypes, compact spindle-like cell morphologies, and rapid growth (Fig. 6a) were available for somatic cell nuclear transfer (SCNT).

\section{Nuclear transfer for generating NRAMP1 knockin cattle}

We randomly picked eight transgenic colonies (evenly split between the WT Cas9 and single Cas9n groups) as donor cells to produce cloned transgenic cows according to the above selection criteria. A total of 2385 and 2434 embryos were successfully reconstructed in vitro (Fig. 6a) for each group. Among these, 525 (22.0\%) and 753 (30.9\%) of the embryos from the WT Cas9 and single

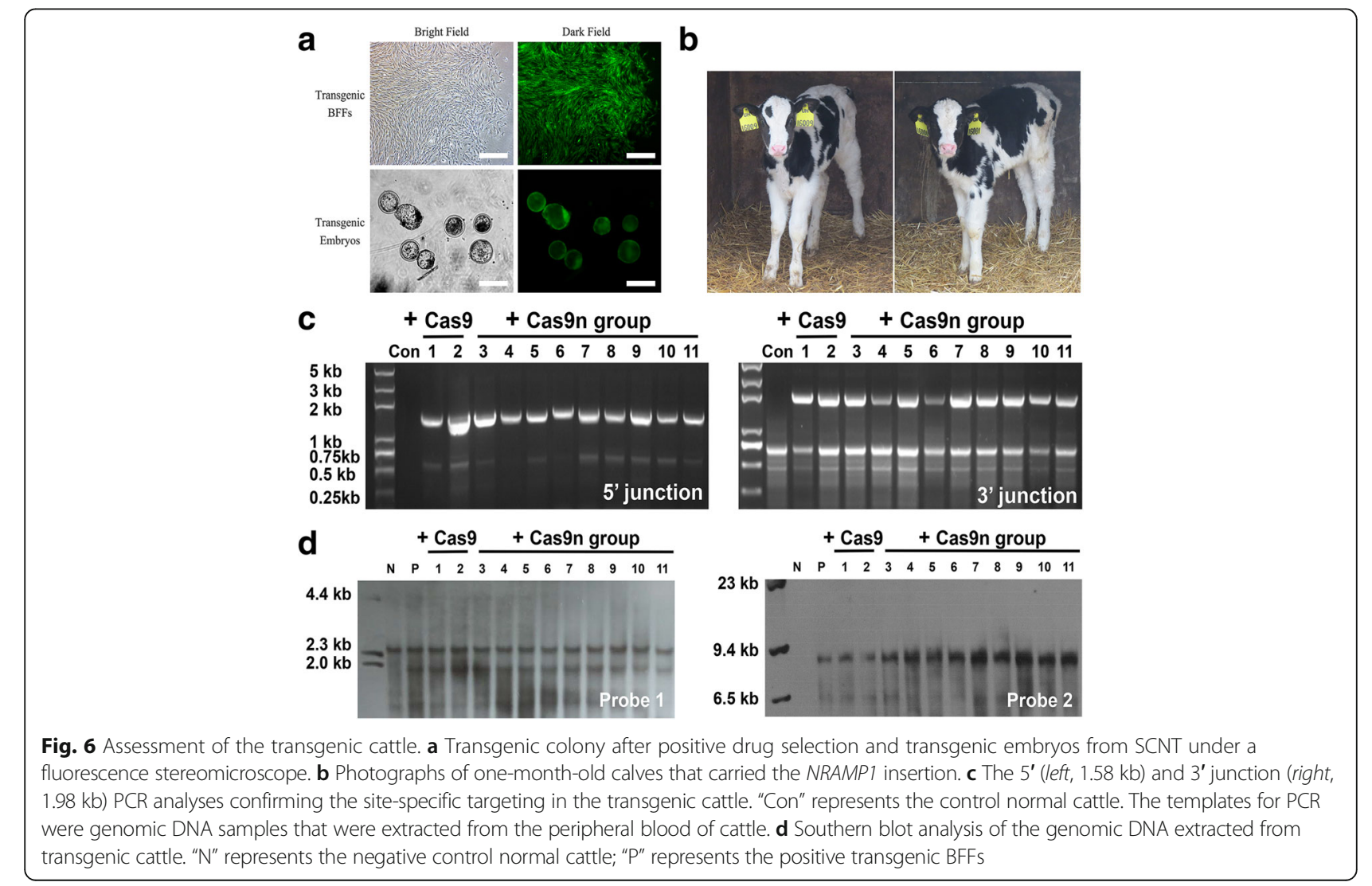


Cas9n groups developed to the blastocyst stage and were transferred into the oviducts of 173 and 248 recipient heifers, respectively. As indicated in Table 1, both the blastocyst formation and pregnancy rates of the Cas9n group were greater than those of the WT Cas9 group, which may be attributable to the less mutagenic NHEJ and low toxicity. Finally, a total of 20 calves (16 from the Cas9n group) were born and 11 calves (nine from the Cas9n group) survived longer than three months (Fig. 6b).

Junction PCR and southern blot analyses were again performed on the remaining 11 living calves (Fig. 6c and d). The results demonstrated that the exogenous NRAMP1 was precisely integrated at target site 45 in a heterozygous form as expected. We also cloned the 15 main sgRNA45 off-target sites designated by ChIP-seq from the transgenic cattle genomes to evaluate the practical off-target effects. Finally, we obtained three indels in the WT Cas9-induced group (Additional file 3: Figure S8), but no typical indels occurred in any of the nine calves from the Cas9n group.

\section{NRAMP1 insertion provides cattle with increased resistance to tuberculosis}

No significant difference was detected in the relative expression levels of the nearby endogenous genes between the transgenic and control cattle based on real-time PCR analysis (Fig. 7a and Additional file 3: Table S7). Furthermore, no NRAMP1 protein was detected in the skin, muscle, heart, liver, lungs, or kidneys of the transgenic cattle by western blot (Fig. 7b). These results indicated that the expression of NRAMP1 was still restricted to dedicated phagocytes as observed in conventional cattle.

To evaluate the biological responses of the transgenic cattle to $M$. bovis infection, we isolated peripheral blood mononuclear cells (PBMCs) from transgenic and normal Holstein-Friesian cows. Subsequently, the PBMCs were induced into macrophages via stimulation with granulocyte macrophage colony-stimulating factor (GM-CSF) for the subsequent experiments as described in Additional file 3: Supplemental Methods and Figure S9. These two groups exhibited similar constitutive expression levels of NRAMP1 and obviously different responses to M. bovis following infection. First, we observed the robust expression of NRAMP1 for the elimination of intracellular mycobacteria in the transgenic groups and the endogenous NRAMP1 protein level also increased after infection (Fig. 7c). Second, the growth rate of M. bovis in the monocyte-derived macrophages (MDMs) from the transgenic cattle was lower than that of the normal group (Fig. 7d). Finally, a notable bias towards the macrophage cell death pathway after infection was observed (Fig. 7e). Flow cytometry analysis revealed that $7.4 \%$ of the challenged macrophages were necrotized in the normal group, whereas the necrosis rate in the transgenic cattle macrophages was $4.2 \%$. Moreover, a nearly twofold increase in the rate of apoptosis after infection was detected in the transgenic cattle compared with the control group (30.2\% $\pm 0.63 \%$ versus $14.9 \% \pm 0.58 \%, P=0.0036$ ).

An in vivo challenge experiment was also performed to further estimate the ability of the transgenic cattle to resist tuberculosis. Six randomly selected transgenic cattle and six experimental control normal cattle (breedmatched, sex-matched, and age-matched with the transgenic cattle) were infected via endobronchial instillation with $5 \times 10^{4} \mathrm{cfu}$ of $M$. bovis [45]. Blood samples were collected at regular intervals throughout the challenge period. Next, IFN- $\gamma$ release assays (IGRAs) were conducted to assess the pathology $[45,46]$. The control cattle developed IFN- $\gamma$ responses within three weeks of stimulation with purified bovine tuberculin protein derivatives (PPD-Bs) and the response continued to steadily increase throughout the 12-week challenge period (Fig. 7f). In contrast, the IFN- $\gamma$ responses against the PPD-Bs in the transgenic cattle were significantly lower than those of the control group after infection (nine weeks: transgenic $0.40 \pm 0.13$ versus control $1.29 \pm 0.12$, $P=0.000 ; 12$ weeks: transgenic $0.36 \pm 0.14$ versus control $1.45 \pm 0.09, P=0.000)$. After the challenge period, we also performed an MTB-specific enzyme-linked immunospot (ELISPOT) assay [47] to confirm our results. As illustrated in Fig. $7 \mathrm{~g}$, the average number of spot-

Table 1 Summary of nuclear transfer results from gene-targeted bovine fetal fibroblast cells

\begin{tabular}{|c|c|c|c|c|c|c|c|c|c|c|}
\hline \multirow[t]{3}{*}{ Nuclear donor } & \multicolumn{5}{|c|}{ Cas9 nuclease } & \multicolumn{5}{|c|}{ Cas9 nickase } \\
\hline & \multicolumn{4}{|c|}{ Typical colonies suitable for SCNT } & \multirow{2}{*}{$\begin{array}{l}\text { Total } \\
-\end{array}$} & \multicolumn{4}{|c|}{ Typical colonies suitable for SCNT } & \multirow{2}{*}{$\begin{array}{l}\text { Total } \\
-\end{array}$} \\
\hline & $45-57$ & $45-86$ & $45-136$ & $45-159$ & & $45-113$ & $45-187$ & $45-243$ & $45-351$ & \\
\hline Embryos obtained & 632 & 578 & 644 & 531 & 2385 & 568 & 674 & 527 & 665 & 2434 \\
\hline Blastocysts (\%) & $137(21.7)$ & $143(24.7)$ & $126(19.6)$ & $119(22.4)$ & $525(22.0)$ & $163(28.7)$ & $210(31.2)$ & $157(29.8)$ & $223(33.5)$ & $753(30.9)$ \\
\hline Recipients & 46 & 49 & 41 & 37 & 173 & 54 & 68 & 51 & 75 & 248 \\
\hline Pregnancies (\%) & $6(13.0)$ & $9(18.4)$ & $2(4.9)$ & $5(13.5)$ & $22(12.7)$ & $9(16.7)$ & $18(26.5)$ & $11(21.6)$ & $23(30.7)$ & $61(24.6)$ \\
\hline Calves at birth & 1 & 2 & 0 & 1 & 4 & 2 & 5 & 3 & 6 & 16 \\
\hline Survived for 3 months & 0 & 1 & 0 & 1 & 2 & 1 & 3 & 2 & 3 & 9 \\
\hline
\end{tabular}



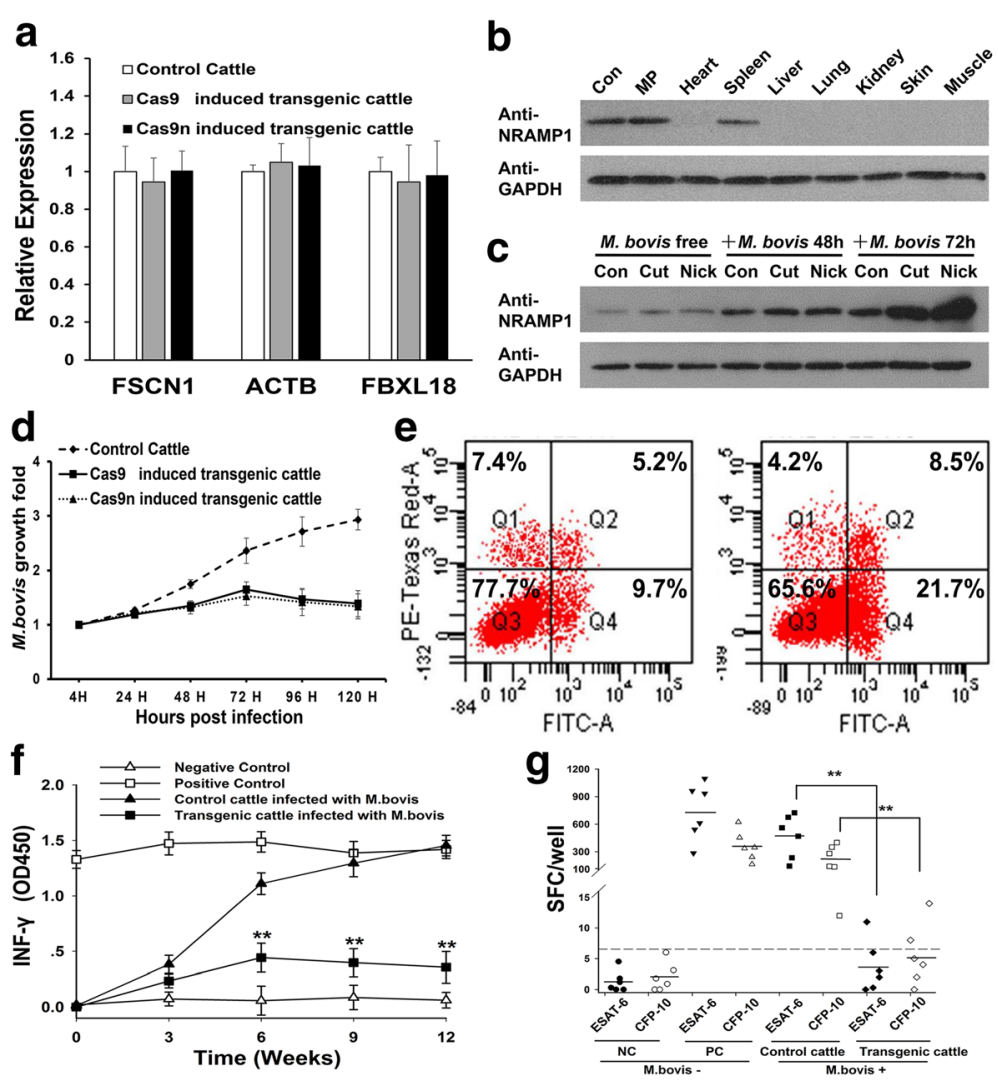

Fig. 7 Assessment of the increased resistance of the transgenic cattle to tuberculosis. a The relative expression levels of the nearby endogenous genes in the F-A locus. Each sample was individually detected in macrophages through real-time PCR, but the data were analyzed according to the group. $\mathbf{b}$ The expression of NRAMP1 was restricted to dedicated phagocytes. The organs were obtained from a pool of dead transgenic cattle. Con, NRAMP1 over-expression Raw264.7 cells; MP macrophages. c The expression of NRAMP1 was highly activated in the transgenic cattle following infection. All the samples were mixed monocyte-derived macrophages (MDMs) that were isolated from the blood of the same group of cattle as a pool. "Con" represents the control normal cattle. $\mathbf{d}$ Multiplication of M. bovis in MDMs from the control or transgenic cattle in vitro. The MDMs were separated from each animal individually and mixed according to group. M. bovis multiplication was determined via cfu assays. e Flow cytometry analysis of the cell death mechanism of the transgenic cattle MDMs after M. bovis infection. Necrotic (Q1), early apoptotic (Q2), and late apoptotic (Q4). Left, infected experiment control MDMs. Right, infected transgenic MDMs. f Amounts of IFN- $\gamma$ produced in the experimental control $(n=6)$ and transgenic $(n=6)$ cattle at regular intervals of 12 weeks. g Concentrations of ESAT-6 and CFP-10 IFN- - -producing SFCs among the PBMCs of the control and transgenic cattle

forming cells (SFC) was significantly lower in the transgenic cattle than the control cattle (early secretory antigenic target-6 (ESAT-6): control $467.8 \pm 235.3$ versus transgenic $3.72 \pm 4.18, P=0.005$; culture filtrate protein10 (CFP-10): control $219.7 \pm 148.1$ versus transgenic 5.5 $\pm 4.96, P=0.016$ ). Moreover, the number of SFCs in the transgenic cattle was not significantly different from that in the negative control cattle (ESAT-6: negative control $1.3 \pm 1.72$ versus transgenic $3.72 \pm 4.18, P=0.219$; CFP10: negative control $1.97 \pm 2.30$ versus transgenic $5.5 \pm$ 4.96, $P=0.145)$. These results indicated that the transgenic cattle exhibited increased resistance to $M$. bovis.

\section{Discussion}

Genome editing has developed rapidly since the seminal discovery of programmable artificial endonucleases such as ZFN, TALEN, and Cas9. These programmable nucleases can produce site-specific DNA DSBs and these
DSBs can enhance the efficiency of HDR by at least two orders of magnitude and/or trigger NHEJ and subsequently lead to targeted mutagenesis [48]. ZFNs and TALENs both require the complex engineering of highly specific DNA-binding domains for proper targeting. In contrast, the CRISPR/Cas9 system only requires a small artificial sgRNA to direct the Cas9 endonuclease to virtually any location in the genome [14]. Due to high efficiency, CRISPR/Cas9 technology can readily simultaneously target several loci including both alleles of the same gene $[7,10,49]$.

However, considering its variable and substantial offtarget effects, specificity remains an issue in this system. To date, no definitive rules are available for predicting Cas9 specificity, but several mutants and corresponding strategies have been proposed to minimize off-target cleavage events. These strategies include the use of double nickase [19, 20], short sgRNAs [50], and a fusion 
protein containing a catalytically inactive $\mathrm{dCas} 9$ and the endonuclease Fok1 $[8,51]$. Because each single indel at an unexpected off-target site may be disastrous, benefits can be derived from these strategies for precise gene therapy and transgenic animal production is possible.

To our knowledge, the current study demonstrates the application of single Cas9n in livestock for the first time. This technique aims to efficiently induce gene insertion at a selected bovine locus and produce transgenic cattle with reduced off-target effects. Moreover, we further demonstrated that the inserted NRAMP1 was correctly expressed and provided cattle with increased resistance to infection with $M$. bovis, which is the mycobacterial pathogen that causes bovine tuberculosis.

Because they have significant influence over targeted specificity, the putative transgenic locus and the specific target site should be selected through meticulous deliberation. Evaluating the practical functions of CRISPR/ Cas9 at the individual sites was a significant task due to the variable mutation rates at the 14 computationally predicted target sites (Fig. 1d). Thus, we performed systematically selective processes, which included cleavage efficiency detection, recombination efficiency detection, and binding specificity mapping, on the selected target site 45 to ensure its quality performance. Moreover, the current study confirmed that this target site can provide the internal transgene with stable and tissue-specific expression (Fig. 7b and c) without disrupting adjacent genes (Figs. 6a, b, and 7a). This finding indicated that the target site 45 of the F-A locus might be a safe harbor for site-specific gene insertion in the bovine genome.

The exact mechanism by which a SSB leads to fewer off-target sites and reduced toxicity targeting compared with a DSB is not yet well understood [52], but this phenomenon is a very active area of research. Generally, the majority of chromosomal single-stranded nicks are rapidly detected and removed by the SSB repair (SSBR) pathway throughout the entire mitotic cycle and no genomic modification occurs during this process [53, 54]. Therefore, single Cas9n-induced HDRs commonly exhibit low efficiencies in some cell types and it has been suggested that this phenomenon should be leveraged for specific gene targeting [5]. Based on the notably high indel rate $(\sim 47 \%)$ of the WT CRISPR/Cas9 at the selected target site 45 , the utilization of a single Cas9ninduced SSB to stimulate HDR with subsequent gene insertion is technically feasible. Additionally, the considerably high efficiencies of the generation of transgenic BFFs $(\sim 18 \%$ positive colony rate) and cattle (9/11 new-born calves) with a single Cas9n group suggests that single Cas9n can be regarded as a valid alternative to the classical WT Cas9 method. Recent studies have focused on improving the specificity of the Cas9 nuclease through rational engineering of the Cas9 protein $[55,56]$.
Considering these tools under development, a single nickase at a selected target site should exhibit excellent performance.

According to our previous studies [43, 44], obtaining homozygous transgene knockin colonies through conducting gene targeting operation once was difficult in BFFs. Although none of the 57 positive colonies gained biallelic NRAMP1 knockin at target site 45 , indels were observed in the other allele in several clones of WT Cas9 group (Additional file 3: Table S6). This finding suggests that Cas9 can cleave both alleles. Hence, obtaining homozygous livestock for propagation is potential for future studies through increasing the amount of transgenic colonies or the use of small molecules and proteins that can increase HDR rates [57, 58].

Regarding the MDMs that were isolated from the transgenic cattle, more robust expression of NRAMP1 was observed following the $M$. bovis challenge compared with the MDMs from the conventional cattle. However, no difference was detected in the constitutive expression levels of NRAMP1 between these groups. The NRAMP1 transgenic cattle exhibited increased resistance to $M$. bovis. This finding might be associated with the activity of the apoptotic pathway after infection, which can reduce the effectiveness of the intracellular survival mechanisms of $M$. bovis [59]. We speculate that the open genomic structure of the inserted NRAMP1 locus might enhance interactions with transcriptional activators, but the specific mechanism by which NRAMP1 determines the fate of macrophages should be further investigated.

\section{Conclusions}

In summary, we demonstrated that a single Cas9n can be used for gene insertion at a selected target site in the cattle genome and that this method is advantageous in terms of avoiding additional indel mutations. The resulting transgenic cattle exhibited increased resistance to $M$. bovis infection. Our study provides an avenue to develop the CRISPR/Cas9 system for agriculture applications.

\section{Methods \\ Construction of Cas9 plasmids}

The query sequence of the F-A locus for the design tool ZiFiT is available in Additional file 1: Supplemental Dataset S1; the 80 potential target sites were inputted into the web-based off-targets predication tool CasOFFinder [29] to evaluate the mismatches number. Through the basic lookup and filter function, we performed statistical analysis of the mismatches number and location of all the 80 sgRNAs, individually. All the data during this procedure are shown in Additional file 2: Supplemental Dataset S2. 
To generate the sgRNA expression construct, we cloned sgRNA into the pSpCas9 (BB) vector for co-expression with Cas9 [30]. Primer sequences for sgRNA cloning are shown in Additional file 3: Table S1. The restriction enzyme BbsI (NEB, UK) and ligation reaction buffer Solution I (Takara, Tokyo, Japan) were used for plasmid construction. The Cas9n with D10A $\mathrm{HNH}^{+} / \mathrm{RuvC}^{-}$mutant plasmid pSpCas9n (BB)-2A-GFP (PX461, Addgene plasmid \# 48140) was also received from Addgene. The catalytically inactive, double mutant dCas9 plasmids were generated by introducing a H840A mutation into the Cas9n plasmid using the QuikChange Site-Directed Mutagenesis kit (Stratagene, CA, USA).

\section{Surveyor nuclease assay}

BFFs were transfected with the DNA constructs described above. Cells were incubated at $37{ }^{\circ} \mathrm{C}$ for $72 \mathrm{~h}$ post transfection prior to genomic DNA extraction. In brief, genomic DNA was extracted using a Universal Genomic DNA Extraction Kit (TaKaRa). The genomic region flanking the CRISPR target locus was PCR amplified using the following primers: Surveyor-detect-F (5'GACTCCTGTAACCTCTGTCCCTG-3') and Surveyordetect-R (5' -TCAGCAGTTGCGGTTCG-3'). The products were purified by PCR Clean-Up Kit (Axygen, CA, USA), digested with Surveyor nuclease (Transgenomic, NE, USA) and analyzed by agarose gel electrophoresis according to the manufacturers' instructions.

Genome modification rates can be estimated first by calculating the relative intensities of digestion products $a, b$, and the undigested band c. The frequency of cutting $f_{\text {cut }}$ is then given by $(\mathrm{a}+\mathrm{b}) /(\mathrm{a}+\mathrm{b}+\mathrm{c})$. The following formula, based on the binomial probability distribution of duplex formation, estimates the percentage of indels in the sample:

$$
\text { Indel } \%=\left(1-\sqrt{\left(1-f_{\text {cut }}\right)}\right) * 100
$$

\section{Cell culture and transfection}

BFFs were isolated from 35-40-day-old fetuses and maintained in DMEM/F12 (Gibco, NY, USA) supplemented with $10 \% \mathrm{FBS}$ at $37{ }^{\circ} \mathrm{C}$ in a $5 \% \mathrm{CO}_{2}$ environment. Raw 264.7 cells (ATCC, VA, USA) were cultured with 1640 (Gibco) supplemented with 10\% FBS (Gibco). BFFs were harvested using $0.25 \%$ trypsin/EDTA solution (Gibco). Cells $\left(1 \times 10^{7}\right)$ were resuspended in Opti-MEM (Gibco), mixed, if not otherwise indicated, with $12 \mu \mathrm{g}$ of linearized donor plasmid and $10 \mu \mathrm{g}$ of Cas9-encoding plasmid, and electroporated at $510 \mathrm{~V}$ with three pulses of 1-ms duration using the BTX Electro-cell manipulator ECM2001 (BTX Technologies, CA, USA). Electroporated cells were sorted via flow cytometry and plated on $10-\mathrm{cm}$ plates at $1 \times 10^{6}$ cells per plate. Individual colonies were selected and expanded after puromycin selection $(2 \mu \mathrm{g} / \mathrm{mL}) 10-14$ days after electroporation.

\section{ChIP experiments and data analysis}

One million cells were seeded to 6 - $\mathrm{cm}$ plates on day 1 , transfected with reformed sgRNAs-plasmids (or dCas9 plasmids without sgRNA) on day 2, and transferred to 10-cm plates on day 3; and cross-linking was done on day 4 approximately about five million cells. Then the transfected cells were subjected to standard ChIP protocol in Pierce Agarose ChIP Kit (Thermo Scientific, NH, USA). The primers used to detect DNA fragments are shown below. For: 5' - TAAGGATTAGAAGGCAGAGTG -3'; Rev: 5' - CCTGTTTTCGTGGAGTTTA $-3^{\prime}$. DNA fragments after ChIP were end-repaired, Atailed, and ligated with indexed adapters. The products were purified and enriched by PCR to create the final cDNA libraries. Target bands were harvested by $2 \%$ agarose gel electrophoresis and quantified by Agilent 2200 . The tagged cDNA libraries were pooled in equal ratios and used for 101-bp paired-end sequencing in a single lane of the Illumina HiSeq 2500 with $51+7$ cycles.

Before read mapping, clean reads were obtained from the raw reads by removing the adaptor sequences, reads with $>5 \%$ ambiguous bases (noted as $\mathrm{N}$ ) and low-quality reads containing more than $20 \%$ of bases with qualities of $<20$. The clean reads were then aligned to B. taurus genome (version: Btau_4.6.1) using the BWA program (0.7.8-r455). For alignment, preliminary experiments were performed to optimize the alignment parameters [32]. We applied the Peak calling between sgRNA and control groups based on the mapped reads utilizing MACS. To eliminate the effect of the Cas9 protein, the peak calling between each sgRNA sample and Venn Analysis between each peak calling result were applied. To analyze sequences in the resulting significant regions that matched the sgRNA guiding sequence and PAM sequence (23 bp), the sgRNA selection tool Cas-Offinder and PATMAN alignment tool [60] were used to align the target sequences to those present under the peak regions. The relationship between the $\mathrm{CpG}$ island and binding site were performed based on the UCSC genome annotation.

\section{Detection of individual colonies by PCR}

Drug-resistant cell clones derived from the transfected cell populations were collected by trypsinization and $70 \%$ of these were plated in serum-containing culture medium and expanded. The remaining clones were resuspended in $20 \mu \mathrm{L}$ of PCR-compatible lysis buffer $(10 \mathrm{mM}$ of Tris- $\mathrm{HCl}, \mathrm{pH} 8.5 ; 50 \mathrm{mM}$ of $\mathrm{KCl} ; 1.5 \mathrm{mM}$ of $\mathrm{MgCl}_{2} ; 0.5 \% \mathrm{NP}-40 ; 0.5 \%$ Tween-20; $400 \mathrm{~g} / \mathrm{mL}$ of proteinase K) for PCR analysis. The lysates were incubated 
at $65{ }^{\circ} \mathrm{C}$ for $60 \mathrm{~min}$ and then at $95^{\circ} \mathrm{C}$ for $15 \mathrm{~min}$. To distinguish the Nramp1-targeted cell clones, $2 \mu \mathrm{L}$ of the DNA lysate was added to a PCR reaction with PCR primers for $5^{\prime}$ junction PCR and subjected to PCR with EmeraldAmp (TaKaRa) using standard methods. Subsequently, 3'-junction PCR was performed on the positive clones to confirm the correct targeting events. The primers used for junction PCR were as follows: $5^{\prime}$-junction, Lj F (5'-AGTTGTG CCCTCCGTGTA-3') and Lj R (5' -CTGCCATGCCCACTCAT-3'); 3'-junction, $\mathrm{Rj} F$ (5'-GCGCATGGCCGAGTT GA-3') and Rj R (5'-CCCGCATTGCTCCCTCT-3').

\section{Southern blot analysis}

Probes for southern blot were amplified from cattle genomic DNA using the following primers: Probe $1, \mathrm{p} 1 \mathrm{~F}\left(5^{\prime}-\mathrm{CCA}\right.$ GTTCTTTGATGGGTGT-3') and p1R (5'-GGCTTGACAGAAGGGTATG-3'); and Probe 2, p2F (5'-AGCAAGC AGGAGACGTGGAA-3') and p2R (5'-CGCTCGTAGAAGGGGAGGTT-3'). PCR products were labeled with digoxigenin using the DIG-High Prime DNA Labeling and Detection Starter Kit II (Roche Diagnostics, IN, USA). Hind III-digested genomic DNA was separated on $1 \%$ (wt/vol) agarose gels, transferred to a nylon membrane (GE Healthcare, USA), and hybridized with 3 '-end digoxigenin-labeled probes. The following procedures were performed using the DIG-High Prime DNA Labeling and Detection Starter Kit II according to the manufacturer's instructions.

\section{Nuclear transfer}

Ovaries were collected from the local abattoir and transported to the laboratory within $4-6 \mathrm{~h}$ in sterile saline at $37{ }^{\circ} \mathrm{C}$. In vitro maturation of oocytes, enucleation, microinjection, and fusion of reconstructed oocytes were carried out in our laboratory according to previously described methods [44]. The reconstructed oocytes were cultured until they developed to the blastocyst stage. Three or four fresh day 7 blastocysts produced in vitro were non-surgically transferred to randomly assigned synchronized recipient heifers on day 7 after estrus. Pregnancy was diagnosed by rectal palpation on day 35 and confirmed by ultrasonography on day 60 after blastocyst transfer.

\section{Cfu assay}

Infection with M. bovis was performed by the State Key Laboratory of Veterinary Etiological Biology (Lanzhou, China). In brief, a bacterial suspension corresponding to a multiplicity of infection (MOI) of 10:1 $\left(\sim 10^{7}\right.$ bacteria per $10^{6}$ cells) was added to the medium and incubated at $37{ }^{\circ} \mathrm{C}$ and $5 \%$ (vol/vol) $\mathrm{CO}_{2}$ for $4 \mathrm{~h}$. Cells were then washed extensively with PBS to remove non-ingested bacteria. At the time points indicated in the text after infection, bacterial cfu were quantitated by plating on
7H10 agar plates (Difco Laboratories, Detroit, Mich) and incubated for eight weeks at $37{ }^{\circ} \mathrm{C}$.

\section{M. bovis challenge experiments}

We had set a control group and an experimental group in the subsequent experiments. Skin tests and IFN- $\gamma$ assays were performed to confirm that cattle were infected with $M$. bovis. The control group comprised a negative control (a normal animal without the transgene or $M$. bovis infection) and a positive control (a normal animal without the transgene but infected with $M$. bovis by endobronchial instillation and diagnosed as tuberculous). The experimental groups comprised the control (a normal animal without the transgene or $M$. bovis infection; breed-matched, sex-matched, and age-matched with the transgenic cattle) and transgenic animals. Positive controls used for the challenge experiment were produced by endobronchial instillation with $5 \times 10^{4} \mathrm{cfu}$ of $M$. bovis. For the challenge experiments, all the calves of experimental groups were infected with $5 \times 10^{4}$ cfu of $M$. bovis (strain AF 2212/97) by endobronchial instillation as previously described [45, 61]. Then blood samples were collected and IFN- $\gamma$ assays were performed to monitor the level of IFN- $\gamma$ release at the time points indicated in Fig. 7f. At the end of the experimental period, the calves were killed by i.v. injection of sodium phenobarbitone.

\section{IGRAs}

IGRAs were performed using a BOVIGAM kit (Prionics AG, Schlieren, Switzerland) according to the manufacturer's instructions. In brief, whole-blood samples were incubated with PPD-B to stimulate the lymphocytes to secrete IFN- $\gamma$. The plasma supernatants were harvested after $24 \mathrm{~h}$ of incubation and IFN- $\gamma$ was estimated using a sandwich enzyme immunoassay. Optical density at $450 \mathrm{~nm}$ was determined using a VICTOR $\times 5$ Multilabel Plate Reader (PerkinElmer, USA).

\section{ELISPOT assay}

MTB-specific ELISPOT assays were performed after the challenge period as previously described [46, 47]. In brief, ELISPOT plates (Millipore, MA, USA) were coated overnight at $4{ }^{\circ} \mathrm{C}$ with mouse anti-bovine IFN- $\gamma$ monoclonal antibody (Thermo Scientific). Peripheral blood mononuclear cells $(\mathrm{PBMCs})\left(2 \times 10^{5}\right)$ then were added and cultured at $37^{\circ} \mathrm{C}$ for $24 \mathrm{~h}$. The cells were stimulated with ESAT-6 and CFP-10 peptides in separate wells following procedures performed strictly according to the manufacturer's recommendations. The response of stimulated cultures was considered positive when the test well contained at least six more spots than the control well. 


\section{Additional files}

Additional file 1: Supplemental dataset S1. (PDF $117 \mathrm{~kb}$ )

Additional file 2: Supplemental dataset S2. (XLSX 8654 kb)

Additional file 3: Supplemental Methods, Figures, and Tables. Supplemental Methods, Figures S1-S9, and Tables S1-S7. (PDF 1638 kb)

\section{Acknowledgments}

We would like to thank Zekun Guo for his expert technical assistance; Mengke Yuan for performing the southern blot analysis; Dai Chen and Qinghua Guo of NovelBio informatics for the ChIP-seq data analysis; and the Keyuan Cloning Company for their assistance in the transgenic cow production.

\section{Funding}

This work was supported by the National Science and Technology Major Project in New Varieties Cultivation of Transgenic Organisms (2016ZX08007-003).

\section{Availability of data and materials}

All data generated or analyzed during this study are included in this article and its supplementary information files. The ChIP-seq data discussed in this paper have been deposited in the NCBI's Gene Expression Omnibus (GEO). The accession number is GSE80376. The bovine ovaries from slaughtered mature cows were collected from the Tumen abattoir, a local abattoir in Xi'An, China. The fetuses and recipient bovine were obtained from Yangling Keyuan Cloning Co. Ltd., China. The plasmids used in this study can be obtained from the corresponding authors on reasonable request.

\section{Authors' contributions}

$Y G, H W$, and $Y Z$. conceived and designed the research; $Y G, Y W, X i n L i u, L C$, and QL performed experiments; YG, CC, Xu Liu, and JZ analyzed the data; $Y G, H W$, and $Y Z$ wrote the manuscript. All authors read and approved the final manuscript.

\section{Competing interests}

The authors declare that they have no competing interests.

\section{Ethics approval}

This study was carried out in strict accordance with the guidelines for the care and use of animals of Northwest A\&F University. All animal experimental procedures were approved by the Animal Care Commission of the College of Veterinary Medicine, Northwest A\&F University. Every effort was made to minimize animal pain, suffering, and distress and to reduce the number of animals used.

Received: 16 August 2016 Accepted: 21 December 2016 Published online: 01 February 2017

\section{References}

1. Filippo JS, Sung P, Klein H. Mechanism of eukaryotic homologous recombination. Annu Rev Biochem. 2008;77:229-57. doi:10.1146/annurev. biochern.77.061306.125255.

2. Meyer M, de Angelis MH, Wurst W, Kuhn R. Gene targeting by homologous recombination in mouse zygotes mediated by zinc-finger nucleases. Proc Natl Acad Sci U S A. 2010;107:15022-6. doi:10.1073/pnas.1009424107.

3. Sommer D, Peters A, Wirtz T, Mai M, Ackermann J, Thabet Y, et al. Efficient genome engineering by targeted homologous recombination in mouse embryos using transcription activator-like effector nucleases. Nat Commun 2014;5:3045. doi:10.1038/Ncomms4045.

4. Hockemeyer D, Wang HY, Kiani S, Lai CS, Gao Q, Cassady JP, et al. Genetic engineering of human pluripotent cells using TALE nucleases. Nat Biotechnol. 2011;29:731-4. doi:10.1038/nbt.1927.

5. Katz SS, Gimble FS, Storici F. To nick or not to nick: comparison of I-scei single- and double-strand break-induced recombination in yeast and human cells. PLoS One. 2014;9:e88840. doi:10.1371/journal.pone.0088840.

6. Cong L, Ran FA, Cox D, Lin SL, Barretto R, Habib N, et al. Multiplex genome engineering using CRISPR/Cas systems. Science. 2013:339:819-23. doi:10. 1126/science.1231143.

7. Yang H, Wang HY, Shivalila CS, Cheng AW, Shi LY, Jaenisch R. One-step generation of mice carrying reporter and conditional alleles by CRISPR/Cas- mediated genome engineering. Cell. 2013;154:1370-9. doi:10.1016/j.cell. 2013.08.022

8. Guilinger JP, Thompson DB, Liu DR. Fusion of catalytically inactive Cas9 to Fokl nuclease improves the specificity of genome modification. Nat Biotechnol. 2014;32:577-82. doi:10.1038/nbt.2909.

9. Shen B, Zhang WS, Zhang J, Zhou JK, Wang JY, Chen L, et al. Efficient genome modification by CRISPR-Cas9 nickase with minimal off-target effects. Nat Methods. 2014;11:399-402. doi:10.1038/nmeth.2857.

10. Niu YY, Shen B, Cui YQ, Chen YC, Wang JY, Wang L, et al. Generation of gene-modified cynomolgus monkey via Cas9/RNA-mediated gene targeting in one-cell embryos. Cell. 2014;156:836-43. doi:10.1016/j.cell.2014.01.027.

11. Galli A, Schiestl RH. Effects of DNA double-strand and single-strand breaks on intrachromosomal recombination events in cell-cycle-arrested yeast cells. Genetics. 1998;149:1235-50.

12. Storici F, Durham CL, Gordenin DA, Resnick MA. Chromosomal site-specific doublestrand breaks are efficiently targeted for repair by oligonucleotides in yeast. Proc Natl Acad Sci U S A. 2003:100:14994-9. doi:10.1073/pnas.2036296100.

13. Lieber MR. The mechanism of double-strand DNA break repair by the nonhomologous DNA end-joining pathway. Annu Rev Biochem. 2010;79: 181-211. doi:10.1146/annurev.biochem.052308.093131.

14. Hsu PD, Scott DA, Weinstein JA, Ran FA, Konermann S, Agarwala V, et al. DNA targeting specificity of RNA-guided Cas9 nucleases. Nat Biotechnol. 2013;31:827-32. doi:10.1038/nbt.2647.

15. Jackson SP, Bartek J. The DNA-damage response in human biology and disease. Nature. 2009;461:1071-8. doi:10.1038/nature08467.

16. Roth JA, Tuggle CK. Livestock models in translational medicine. ILAR J. 2015; 56:1-6. doi:10.1093/ilar/ilv011.

17. Pattanayak V, Lin S, Guilinger JP, Ma EB, Doudna JA, Liu DR. High-throughput profiling of off-target DNA cleavage reveals RNA-programmed Cas9 nuclease specificity. Nat Biotechnol. 2013;31:839-43. doi:10.1038/nbt.2673.

18. Cho SW, Kim S, Kim Y, Kweon J, Kim HS, Bae S, et al. Analysis of off-target effects of CRISPR/Cas-derived RNA-guided endonucleases and nickases. Genome Res. 2014;24:132-41. doi:10.1101/gr.162339.113.

19. Mali P, Aach J, Stranges PB, Esvelt KM, Moosburner M, Kosuri S, et al. CAS9 transcriptional activators for target specificity screening and paired nickases for cooperative genome engineering. Nat Biotechnol. 2013;31:833-8. doi:10.1038/nbt.2675.

20. Ran FA, Hsu PD, Lin CY, Gootenberg JS, Konermann S, Trevino AE, et al. Double nicking by RNA-guided CRISPR Cas9 for enhanced genome editing specificity. Cell. 2013;154:1380-9. doi:10.1016/j.cell.2013.08.021.

21. Sander JD, Zaback P, Joung JK, Voytas DF, Dobbs D. Zinc Finger Targeter (ZiFiT): An engineered zinc finger/target site design tool. Nucleic Acids Res. 2007;35:W599-605. doi:10.1093/nar/gkm349.

22. Sander JD, Maeder ML, Reyon D, Voytas DF, Joung JK, Dobbs D. ZiFiT (Zinc Finger Targeter): an updated zinc finger engineering tool. Nucleic Acids Res. 2010;38:W462-W8. doi:10.1093/nar/gkq319.

23. Mali P, Yang LH, Esvelt KM, Aach J, Guell M, DiCarlo JE, et al. RNA-guided human genome engineering via Cas9. Science. 2013;339:823-6. doi:10.1126/ science.1232033.

24. Kim H, Kim JS. A guide to genome engineering with programmable nucleases. Nat Rev Genet. 2014;15:321-34. doi:10.1038/nrg3686.

25. Fu YF, Foden JA, Khayter C, Maeder ML, Reyon D, Joung JK, et al. Highfrequency off-target mutagenesis induced by CRISPR-Cas nucleases in human cells. Nat Biotechnol. 2013;31:822-6. doi:10.1038/nbt.2623.

26. Wu XB, Scott DA, Kriz AJ, Chiu AC, Hsu PD, Dadon DB, et al. Genome-wide binding of the CRISPR endonuclease Cas9 in mammalian cells. Nat Biotechnol. 2014:32:670-6. doi:10.1038/nbt.2889.

27. Jiang WY, Bikard D, Cox D, Zhang F, Marraffini LA. RNA-guided editing of bacterial genomes using CRISPR-Cas systems. Nat Biotechnol. 2013;31:233-9. doi:10.1038/nbt.2508.

28. Kuscu C, Arslan S, Singh R, Thorpe J, Adli M. Genome-wide analysis reveals characteristics of off-target sites bound by the Cas9 endonuclease. Nat Biotechnol. 2014;32:677-83. doi:10.1038/nbt.2916.

29. Bae S, Kweon J, Kim HS, Kim JS. Microhomology-based choice of Cas9 nuclease target sites. Nat Methods. 2014;11:705-6. doi:10.1038/nmeth.3015.

30. Ran FA, Hsu PD, Wright J, Agarwala V, Scott DA, Zhang F. Genome engineering using the CRISPR-Cas9 system. Nat Protoc. 2013:8:2281-308. doi:10.1038/nprot.2013.143

31. Guschin DY, Waite AJ, Katibah GE, Miller JC, Holmes MC, Rebar EJ. A rapid and general assay for monitoring endogenous gene modification. Methods Mol Biol. 2010;649:247-56. doi:10.1007/978-1-60761-753-2_15. 
32. Li H, Durbin R. Fast and accurate short read alignment with BurrowsWheeler transform. Bioinformatics. 2009;25:1754-60. doi:10.1093/ bioinformatics/btp324.

33. Zhang $Y$, Liu T, Meyer CA, Eeckhoute J, Johnson DS, Bernstein BE, et al. Model-based analysis of ChIP-Seq (MACS). Genome Biol. 2008;9:R137. doi:10.1186/gb-2008-9-9-r137.

34. Poorey K, Viswanathan R, Carver MN, Karpova TS, Cirimotich SM, McNally JG, et al. Measuring chromatin interaction dynamics on the second time scale at single-copy genes. Science. 2013;342:369-72. doi:10.1126/science.1242369.

35. Vidal S, Tremblay ML, Govoni G, Gauthier S, Sebastiani G, Malo D, et al. The Ity/Lsh/Bcg locus - natural-resistance to infection with intracellular parasites is abrogated by disruption of the Nramp1 gene. J Exp Med. 1995;182:655-66. doi:10.1084/jem.182.3.655.

36. Barthel R, Feng JW, Piedrahita JA, McMurray DN, Templeton JW, Adams LG. Stable transfection of the bovine NRAMP1 gene into murine RAW264.7 cells: effect on Brucella abortus survival. Infect Immun. 2001;69:3110-9. doi: 10.1128/lai.69.5.3110-3119.2001.

37. Zaharik ML, Cullen VL, Fung AM, Libby SJ, Choy SLK, Coburn B, et al. The Salmonella enterica serovar typhimurium divalent cation transport systems $\mathrm{MntH}$ and SitABCD are essential for virulence in an Nramp1(G169) murine typhoid model. Infect Immun. 2004;72:5522-5. doi:10.1128/lai.72.9.5522-5525.2004.

38. Li HT, Zhang TT, Zhou YQ, Huang QH, Huang J. SLC11A1 (formerly NRAMP1) gene polymorphisms and tuberculosis susceptibility: a meta-analysis. Int J Tuberc Lung Dis. 2006;10:3-12.

39. Hedges JF, Kimmel E, Snyder DT, Jerome M, Jutila MA. Solute carrier 11A1 is expressed by innate lymphocytes and augments their activation. J Immunol. 2013;190:4263-73. doi:10.4049/jimmunol.1200732.

40. Cheng $X$, Wang HY. Multiple targeting motifs direct NRAMP1 into lysosomes. Biochem Biophys Res Commun. 2012;419:578-83. doi:10.1016/j. bbrc.2012.02.078.

41. Kim JH, Lee SR, Li LH, Park HJ, Park JH, Lee KY, et al. High cleavage efficiency of a 2A peptide derived from porcine Teschovirus-1 in human cell lines, zebrafish and mice. PLoS One. 2011;6:e18556. doi:10.1371/journal. pone.0018556.

42. Yu Y, Tong Q, Li ZX, Tian JH, Wang YZ, Su F, et al. Improved site-specific recombinase-based method to produce selectable marker- and vectorbackbone-free transgenic cells. Sci Rep. 2014;4:4240. doi:10.1038/Srep04240.

43. Wu HB, Wang YS, Zhang Y, Yang MQ, LV JX, Liu J, et al. TALE nickase-mediated SP110 knockin endows cattle with increased resistance to tuberculosis. Proc Natl Acad Sci U S A. 2015;112:E1530-E9. doi:10.1073/pnas.1421587112.

44. Liu X, Wang YS, Guo WJ, Chang BH, Liu J, Guo ZK, et al. Zinc-finger nickasemediated insertion of the lysostaphin gene into the beta-casein locus in cloned cows. Nat Commun. 2013;4:2565. doi:10.1038/Ncomms3565.

45. Vordermeier HM, Chambers MA, Cockle PJ, Whelan AO, Simmons J, Hewinson RG. Correlation of ESAT-6-specific gamma interferon production with pathology in cattle following Mycobacterium bovis BCG vaccination against experimental bovine tuberculosis. Infect Immun. 2002;70:3026-32. doi:10.1128/lai.70.6.3026-3032.2002.

46. Flynn JL, Gideon HP, Mattila J, Lin PL. Immunology studies in non-human primate models of tuberculosis. Immunol Rev. 2015;264:60-73. doi:10.1111/imr.12258.

47. Khader SA, Bell GK, Pearl JE, Fountain JJ, Rangel-Moreno J, Cilley GE, et al. $\mathrm{IL}-23$ and $\mathrm{IL}-17$ in the establishment of protective pulmonary CD4(+) T cell responses after vaccination and during Mycobacterium tuberculosis challenge. Nat Immunol. 2007;8:369-77. doi:10.1038/ni1449.

48. Bibikova M, Golic M, Golic KG, Carroll D. Targeted chromosomal cleavage and mutagenesis in Drosophila using zinc-finger nucleases. Genetics. 2002;161:1169-75.

49. Li W, Teng F, Li TD, Zhou Q. Simultaneous generation and germline transmission of multiple gene mutations in rat using CRISPR-Cas systems. Nat Biotechnol. 2013;31:684-6. doi:10.1038/nbt.2652.

50. Fu YF, Sander JD, Reyon D, Cascio VM, Joung JK. Improving CRISPR-Cas nuclease specificity using truncated guide RNAs. Nat Biotechnol. 2014;32: 279-84. doi:10.1038/nbt.2808.

51. Wyvekens N, Topkar W, Khayter C, Joung JK, Tsai SQ. Dimeric CRISPR RNA-guided Fokl-dCas9 nucleases directed by truncated gRNAs for highly specific genome editing. Hum Gene Ther. 2015;26:425-31. doi:10.1089/hum. 2015.084 .

52. Metzger MJ, McConnell-Smith A, Stoddard BL, Miller AD. Single-strand nicks induce homologous recombination with less toxicity than double-strand breaks using an AAV vector template. Nucleic Acids Res. 2011;39:926-35. doi:10.1093/nar/gkq826.
53. el-Khamisy SF, Caldecott KW. DNA single-strand break repair and spinocerebellar ataxia with axonal neuropathy-1. Neuroscience. 2007;145: 1260-6. doi:10.1016/j.neuroscience.2006.08.048.

54. Caldecott KW. Single-strand break repair and genetic disease. Nat Rev Genet. 2008;9:619-31. doi:10.1038/nrg2380.

55. Kleinstiver BP, Pattanayak V, Prew MS, Tsai SQ, Nguyen NT, Zheng Z, et al. High-fidelity CRISPR-Cas9 nucleases with no detectable genome-wide off-target effects. Nature. 2016;529:490-5. doi:10.1038/nature16526.

56. Slaymaker IM, Gao L, Zetsche B, Scott DA, Yan WX, Zhang F. Rationally engineered Cas9 nucleases with improved specificity. Science. 2016;351:84-8. doi:10.1126/science.aad5227.

57. Maruyama T, Dougan SK, Truttmann MC, Bilate AM, Ingram JR, Ploegh HL. Increasing the efficiency of precise genome editing with CRISPR-Cas9 by inhibition of nonhomologous end joining. Nat Biotechnol. 2015;33:538-42. doi:10.1038/nbt.3190.

58. Chu VT, Weber T, Wefers B, Wurst W, Sander S, Rajewsky K, et al. Increasing the efficiency of homology-directed repair for CRISPR-Cas9-induced precise gene editing in mammalian cells. Nat Biotechnol. 2015;33:543-8. doi:10.1038/nbt.3198.

59. Tuggle CK, Waters WR. Tuberculosis-resistant transgenic cattle. Proc Natl Acad Sci U S A. 2015;112:3854-5. doi:10.1073/pnas.1502972112.

60. Prufer K, Stenzel U, Dannemann M, Green RE, Lachmann M, Kelso J. PatMaN: rapid alignment of short sequences to large databases. Bioinformatics. 2008; 24:1530-1.

61. Vordermeier HM, Cockle PC, Whelan A, Rhodes S, Palmer N, Bakker D, et al. Development of diagnostic reagents to differentiate between Mycobacterium bovis BCG vaccination and M-bovis infection in cattle. Clin Diagn Lab Immunol. 1999;6:675-82.

\section{Submit your next manuscript to BioMed Central and we will help you at every step:}

- We accept pre-submission inquiries

- Our selector tool helps you to find the most relevant journal

- We provide round the clock customer support

- Convenient online submission

- Thorough peer review

- Inclusion in PubMed and all major indexing services

- Maximum visibility for your research

Submit your manuscript at www.biomedcentral.com/submit
C Biomed Central 